\title{
Simple polynomial classes of chaotic jerky dynamics
}

\author{
Ralf Eichhorn *, Stefan J. Linz, Peter Hänggi \\ Theoretische Physik I, Institut für Physik, Universität Augsburg, D-86135 Augsburg, Germany
}

Accepted 18 October 2000

\begin{abstract}
Third-order explicit autonomous differential equations, commonly called jerky dynamics, constitute a powerful approach to understand the properties of functionally very simple but nonlinear three-dimensional dynamical systems that can exhibit chaotic longtime behavior. In this paper, we investigate the dynamics that can be generated by the two simplest polynomial jerky dynamics that, up to these days, are known to show chaotic behavior in some parameter range. After deriving several analytical properties of these systems, we systematically determine the dependence of the long-time dynamical behavior on the system parameters by numerical evaluation of Lyapunov spectra. Some features of the systems that are related to the dependence on initial conditions are also addressed. The observed dynamical complexity of the two systems is discussed in connection with the existence of homoclinic orbits. (C) 2001 Elsevier Science Ltd. All rights reserved.
\end{abstract}

\section{Introduction}

It is well known [1-8] that chaotic behavior in time-continuous, autonomous dynamical systems requires a phase-space dimension which is equal to three or larger and nonlinearities in the model equations. It is, however, not definitely known, not even for the simplest case of three-dimensional phase-spaces, what degree of nonlinearity is necessary for the creation of chaos. So, it is tempting to pose the following question: What are the most elementary functional forms of three-dimensional dynamical systems that do exhibit chaotic behavior?

For polynomial vector fields, this problem has been attacked in several recent publications and seems to be related not only to the number and degree of nonlinear monomials but also to the total number of appearing terms. Zhang and Heidel $[9,10]$ have investigated the possibility of chaotic dynamics in systems with only quadratic nonlinearities. They found out that quadratic systems with a total of less than five terms on the right-hand side of the corresponding three first-order differential equations cannot exhibit chaotic behavior. ${ }^{1}$ Their work was motivated by former studies of Sprott [11,12]. Sprott has used a computer search procedure to find systems that are functionally as simple as possible but nevertheless chaotic [11]. He was able to identify 19 distinct chaotic models with vector fields that consist of five terms with two quadratic nonlinearities or of six terms with one quadratic nonlinearity. In subsequent studies [12,13], Sprott even reported a chaotic model with a total of only five terms where one is a quadratic nonlinearity. All these models are algebraically simpler than the prominent models of Lorenz [14] and Rössler [15].

\footnotetext{
${ }^{*}$ Corresponding author. Tel.: +49-821-598-3230; fax: +49-821-598-3222.

E-mail address: ralf.eichhorn@physik.uni-augsburg.de (R. Eichhorn).

${ }^{1}$ More precisely: for dissipative systems, this statement is proven rigorously [9], whereas in the conservative case it is proven only for almost all systems [10]. The lone exception, $\dddot{z}=\dot{z}^{2}-z^{2}$, appears numerically to have a unique unstable periodic orbit with all other solutions being unbounded [10].
} 
A further simplification was achieved in a recent study [16] by transforming the Sprott models to explicit third-order ordinary differential equations, so-called jerky dynamics [17-24],

$$
\dddot{x}=J(x, \dot{x}, \ddot{x}),
$$

where the overdots represent the derivatives with respect to time $t$. This constitutes a simplification in the following sense. Defining $\dot{x}=y$ and $\ddot{x}=z$, any jerky dynamics can obviously be rewritten as a dynamical system [25]

$$
\dot{x}=y, \quad \dot{y}=z, \quad \dot{z}=J(x, y, z) .
$$

The first and second component of this system are algebraically very simple (in fact are the simplest ones for an effectively three-dimensional dynamical system) and are identical for all jerky dynamics. The third component consists of the, in general, nonlinear jerk function $J(x, y, z)$. After transforming a threedimensional dynamical system to a jerky dynamics, solely the number and types of terms appearing in the jerk function reflect the complexity of the linear and nonlinear couplings between the different variables of the original dynamical system. In this sense, one can understand the result that different dynamical systems with the same total of terms and nonlinearities can lead to jerky dynamics with different numbers of terms and nonlinearities [16]. Therefore, the functional complexity of different dynamical systems can be compared more directly and more clearly if these systems are recast as jerky dynamics. From this point of view, functionally elementary jerky dynamics, that exhibit chaos, also constitute the simplest chaotic threedimensional dynamical systems.

Apart from this, the representation of dynamical systems as jerky dynamics has another useful and important advantage: based on their jerky dynamics, a classification of different dynamical systems is possible, because the transformation of certain functionally different three-dimensional dynamical systems can lead to the same jerky dynamics. In [16], it has been shown that 16 of the 19 simple chaotic Sprott models [11] and the toroidal model of Rössler [26] can be classified into seven distinct basic classes of jerky dynamics (labeled by $\mathrm{JD}_{1}-\mathrm{JD}_{7}$ ) with different number and types of quadratic nonlinearities. The two simplest resulting classes of jerky dynamics are $\mathrm{JD}_{1}$, i.e.,

$$
\dddot{x}=k_{1} \ddot{x}+k_{2} x+x \dot{x}+k_{3},
$$

and $\mathrm{JD}_{2}$, i.e.,

$$
\dddot{x}=k_{1} \ddot{x}+k_{2} \dot{x}+x^{2}+k_{3},
$$

possessing only one constant, two linear and one quadratic term in their jerk functions $J(x, \dot{x}, \ddot{x})$. These two classes alone contain nine of the original models. Moreover, also Sprott's simplest model which has already been given as a jerky dynamics [12,13] fits into these classes. It belongs to $\mathrm{JD}_{1}$ as the special case where the constant term $k_{3}$ is zero. The model $\mathrm{JD}_{2}$ has been introduced for the first time in the form $\ddot{x}+\beta \dot{x}+x=\eta$, $\dot{\eta}=\mu x(1-x)$ as a forced oscillator that displays a transition to chaotic behavior for appropriately chosen parameter values [27].

The jerk classes $\mathrm{JD}_{1}$ and $\mathrm{JD}_{2}$, Eqs. (3) and (4), are the algebraically simplest polynomial classes of dynamical systems that are known to exhibit chaotic behavior. Therefore, a detailed study of the dynamical properties of these two systems is interesting and important. In the slightly different functional form $\dddot{x}+\ddot{x}+b \dot{x}-c x+x^{2}=0$ that can be obtained from Eq. (4) by a translation of the origin, the class $\mathrm{JD}_{2}$ has already been investigated as an example for the complicated local bifurcation structure that appears in the neighborhood of homoclinic orbits at a fixed point of saddle-focus type and its consequences for global bifurcation schemes near the homoclinicity including the occurrence of chaos [28-30]. Here, however, we are mainly interested in a global picture of the dynamical properties of the two classes $\mathrm{JD}_{1}$ and $\mathrm{JD}_{2}$, rather than in detailed local analysis of very small parameter ranges. Specifically, we want to detect the distinct regions of the parameter space with different character of the long-time dynamics, i.e., where the dynamics approaches a fixed point, a limit cycle, a chaotic strange attractor, or diverges. The interpretation of our results, though, will bring us back to the investigations of Glendinning and Sparrow [29] and Arnéodo et al. [30]. 
Several different points in the parameter space of $\mathrm{JD}_{1}$ and $\mathrm{JD}_{2}$ with chaotic dynamics (for specific initial values) are already known [16,27-30]. In [16], they were obtained from the original models $[11,12,26]$ that belong to the corresponding basic class of jerky dynamics. The predominantly numerical study presented here will complete this very fragmentary picture. In this context, we also address the dependence of the dynamical behavior on initial conditions. Since the two classes $\mathrm{JD}_{1}$ and $\mathrm{JD}_{2}$ are derived from several different three-dimensional dynamical systems by globally invertible transformations [16], the understanding of the dynamical behavior of $\mathrm{JD}_{1}$ or $\mathrm{JD}_{2}$, respectively, simultaneously covers the corresponding dynamics of the original models by Sprott [11,12] and Rössler [26].

This paper is organized as follows. Section 2 is devoted to a general discussion of several analytical methods that will be used later on to derive some properties of the investigated jerky dynamics. Then, these found properties are utilized to focus the numerics on the interesting regions in the parameter space. The reasoning behind the numerical methods are also described in Section 2. In Section 3, the jerk class $\mathrm{JD}_{1}$ is studied in detail by applying the analytical and numerical tools of Section 2. The same procedure is applied to the class $\mathrm{JD}_{2}$ in Section 4. Section 5 contains the summary and discussion of our results.

\section{Analytical and numerical tools}

The investigation of the jerk classes $\mathrm{JD}_{1}$ and $\mathrm{JD}_{2}$ is split into two parts. First, we use several analytical methods to confine the regions of the parameter spaces where potentially interesting dynamics can occur, i.e., where the dynamics is not divergent. These analytical methods are the stability analysis of fixed points, the Hopf-bifurcation, the time-evolution of a volume in phase-space and a no-chaos theorem introduced in [16]. Second, we use a numerical approach to detect different types of long-time dynamics and their dependence on the parameters that enter into the jerk system in question. These numerical studies are based on the concept of Lyapunov exponents. In the following, we shortly describe all these methods and concepts in a general way without referring to a specific jerky dynamics.

(i) Stability analysis of fixed points and Hopf-bifurcation. Given a jerky dynamics (1) (or, equivalently, recast in the form (2) of a dynamical system) we assume that it has a fixed point at $x^{*} \in \mathbb{R}, \dot{x}^{*}=0, \ddot{x}^{*}=0$, such that $J\left(x^{*}, 0,0\right)=0$. Linearizing the jerky dynamics around this fixed point by setting $x(t)=x^{*}+\varepsilon(t)$ and using the ansatz $\varepsilon(t) \sim \mathrm{e}^{\lambda t}$ for the small perturbation $\varepsilon(t)$, one obtains a characteristic equation of the form

$$
\lambda^{3}-T \lambda^{2}-K \lambda-D=0 .
$$

The real-valued quantities $T, K$ and $D$ contain the parameters of the underlying jerky dynamics. From the criterion of Routh-Hurwitz [31] follows that the fixed point is stable (i.e., the real parts of all roots $\lambda_{i}$ $(i=1,2,3)$ of Eq. (5) are negative) only if the conditions

$$
T<0, \quad K<0, \quad D<0, \quad T K+D>0
$$

are fulfilled. For $T K=-D(T, K, D<0)$, the fixed point becomes unstable and the two complex roots of (5) cross the imaginary axes (of the complex $\lambda$-plane), while the third root remains real and negative. This can be seen by inserting $\lambda=\mathrm{i} \omega$ ( $\omega$ real) into Eq. (5) and comparing imaginary and real parts. Therefore, at $T K=-D(T, K, D<0)$ generically a limit cycle arises via a Hopf-bifurcation [7].

(ii) Time-evolution of phase-space volumes. The volume contraction rate of a (three-dimensional) dynamical system $\dot{\mathbf{x}}=\mathbf{F}(\mathbf{x})$ is given by $\Lambda=\nabla \cdot \mathbf{F}$. If $\Lambda$ is constant, the time-evolution of a volume $V(t)$ in phase-space is described by $V(t)=V(t=0) \mathrm{e}^{\Lambda t}[1,3]$. For negative $\Lambda$, any phase-space volume shrinks exponentially fast; the dynamical system is dissipative and can have stable attractors. If $\Lambda=0$ holds, phase-space-volumes are conserved and the dynamical system is called conservative. For positive $\Lambda$, however, the volumes expand and there are only unstable fixed points or limit cycles or possibly chaotic repellors. Therefore, the dynamics diverges for $t \rightarrow \infty$ if the initial value does not lie exactly at one of such unstable sets (which will almost never be the case for arbitrarily chosen initial values). The volume contraction rate of a jerky dynamics (1) is obtained from its representation as a dynamical system (cf. Eq. (2)) 


$$
\Lambda=\partial_{\ddot{x}} J(x, \dot{x}, \ddot{x}) .
$$

From the above discussion follows that one can only expect bounded dynamical behavior in a dynamical system or a jerky dynamics, if $\Lambda \leqslant 0$ is valid.

(iii) No-chaos theorem. In [16], a theorem that excludes actual three-dimensional dynamical behavior and, therefore, in particular chaotic oscillations for certain parameter regions of a jerky dynamics (and even for some classes of jerky dynamics) has been proven. The following special case of this theorem is needed here: a jerky dynamics (1) that can be written as

$$
\ddot{x}+\Omega(x, \dot{x})=\int^{t} f(x(\tau)) \mathrm{d} \tau,
$$

with $f$ being either a positive or a negative semidefinite function for all $x$ cannot show chaotic behavior. If, moreover, $f$ is of the form $f(x)=\widetilde{f}(x)+c$ with a positive (negative) constant $c \in \mathbb{R}$ and a positive (negative) semidefinite function $\widetilde{f}$, the dynamics eventually diverges for all initial values (except for those that coincide with fixed points of (8)).

(iv) Lyapunov exponents. The Lyapunov exponents of a dynamical system [1] constitute a measure for the exponential divergence (or convergence) of initially nearby trajectories. For a mathematically rigorous definition, we refer to [32] and references therein. The property of Lyapunov exponents, which is important for our problem, is the following: the type of an attractor that is approached in the long-time limit is characterized by the spectrum of the corresponding Lyapunov exponents [32,33]. For phase-space dimension three, e.g., an attracting fixed point possesses the Lyapunov spectrum $\{-,-,-\}$, an attracting limit cycle $\{0,-,-\}$, an attracting 2-torus $\{0,0,-\}$ and a chaotic attractor $\{+, 0,-\}[1,32]$, where + denotes a positive, - a negative and 0 a zero Lyapunov exponent. Therefore, to determine the long-time behavior of a jerky dynamics, i.e., the type of the attractor, we must numerically compute the set of all Lyapunov exponents. In doing so, we use the algorithm that traces back to the works of Shimada and Nagashima [34] and Benettin et al. [35], as reviewed in detail in [1].

\section{The class $\mathbf{J D}_{1}$}

Eq. (3) possesses one single fixed point (for $k_{2} \neq 0$ ) given by $x^{*}=-k_{3} / k_{2}, \dot{x}^{*}=0, \ddot{x}^{*}=0$. The position of this fixed point depends on the specific values of the parameters $k_{2}$ and $k_{3}$. For practical purposes, however, it is advantageous that the location of the fixed point is independent of any parameter value. Moreover, until now, only the possibility of rescaling the dependent variable $x$ has been used [16] to set the coefficient of the quadratic term to +1 . By additionally rescaling the time $t$, one can reduce the number of free parameters further from three to two. Then, the parameter space is just a plane and can easily be visualized. However, it has to be taken into account that the direction of time must not be reversed by this procedure.

Therefore, for an analysis of the class $\mathrm{JD}_{1}$, we first recast the dynamics in a more convenient form. Then, using the methods of Section 2 we analytically derive some simple but important properties of the jerk class $\mathrm{JD}_{1}$.

\subsection{Transformation and analytical properties}

To "fix" the fixed point and to rescale time we use the transformation

$$
\bar{x}=A x+B, \quad \bar{t}=C t \quad(C>0)
$$

for $x$ and $t$ yielding the new quantities $\bar{x}$ and $\bar{t}$. Choosing

$$
A=\frac{1}{k_{1}^{2}}, \quad B=\frac{k_{3}}{k_{1}^{2} k_{2}}, \quad C=\left|k_{1}\right| \quad\left(k_{1}, k_{2} \neq 0\right),
$$


and introducing

$$
a=\frac{k_{3}}{k_{1}^{2} k_{2}}, \quad b=-\frac{k_{2}}{\left|k_{1}\right|^{3}},
$$

from (3) one obtains

$$
\dddot{x}= \pm \ddot{x}-a \dot{x}-b x+x \dot{x} .
$$

Here and in the following, we have dropped the overbars, i.e., we have used again the more convenient notation $x$ and $t$ instead. Now, according to Eq. (11), Sprott's minimal jerk model $[12,13]\left(k_{3}=0\right.$ in $\left.(3)\right)$ is obtained by setting $a=0$.

With the choice of the transformation parameters in Eq. (10) we have excluded the cases that $k_{1}$ or $k_{2}$ are zero. These, however, are not essential restrictions. From the no-chaos theorem discussed in Section 2, we infer that for $k_{2}=0$ the dynamics of $\mathrm{JD}_{1}$, Eq. (3), always diverges and, therefore, need not be considered. For $k_{1}=0, \mathrm{JD}_{1}$ becomes conservative. Since we restrict ourselves to dissipative dynamics, this special case of $\mathrm{JD}_{1}$ will not be studied here.

The distinction between the two signs of the $\ddot{x}$ term in Eq. (12) is due to the choice of the time-scaling factor $C$ as the modulus of $k_{1}$; the plus sign is valid for positive $k_{1}$, the minus sign for negative $k_{1}$. These signs, however, determine the volume contraction rate of $\mathrm{JD}_{1}$ (cf. Section 2) and we find $\Lambda= \pm 1$. It follows that $\mathrm{JD}_{1}$ can have stable attractors only for the minus sign of the $\ddot{x}$ term, whereas for the plus sign the dynamics is divergent or located on an unstable set in phase-space. Therefore, the form of $\mathrm{JD}_{1}$ with possibly interesting, i.e., bounded, dynamics reads

$$
\dddot{x}=-\ddot{x}-a \dot{x}-b x+x \dot{x} .
$$

The completely unstable sets that might exist for the plus sign of the $\ddot{x}$ term can be found by making them stable via time-reversal. Here, we can make use of the following symmetry property of $\mathrm{JD}_{1}$. The transformations $t \rightarrow-t$ and $b \rightarrow-b$ convert the equation $\dddot{x}=+\ddot{x}-a \dot{x}-b x+x \dot{x}$ to Eq. (13). Therefore, the attractors of (13) constitute the repelling sets of $\dddot{x}=+\ddot{x}-a \dot{x}-b x+x \dot{x}$ for sign-inverted parameter $b$.

The fixed point of Eq. (13) is located at $x^{*}=0, \dot{x}^{*}=0, \ddot{x}^{*}=0$, independent of the parameters $a$ and $b$. Its stability properties can be determined by using the procedure shortly described in Section 2. One obtains a characteristic equation (5) with coefficients

$$
T=-1, \quad K=-a, \quad D=-b .
$$

It follows that the fixed point is stable for $a>0, b>0$ and $a>b$ (cf. Eq. (6)) and that a limit cycle arises at the line $a=b$ (for $a, b>0$ ).

Summarizing the results of this Section, we find the following: the class $\mathrm{JD}_{1}$ is completely represented by Eq. (13) which has only a two-dimensional parameter space. This equation, therefore, constitutes the starting point for the further numerical study of $\mathrm{JD}_{1}$, where the region of the parameter space with positive $a$ and $b$ and $b>a$ is the most interesting one.

\subsection{Numerical results}

To investigate the dependence of the long-time dynamics of $\mathrm{JD}_{1}$ on different values of the parameters $a$ and $b$ we have computed the set of all Lyapunov exponents (cf. Section 2) of Eq. (13). ${ }^{2}$ The calculations have been performed for the initial values $x(t=0)=0.04, \dot{x}(t=0)=0.02$ and $\ddot{x}(t=0)=0.0$ which are close to the fixed point of $\mathrm{JD}_{1}$. Here, the part of the parameter space $(a, b)$ has been considered that is given by $a \in[-2.0,+2.0]$ and $b \in[-2.0,+2.0]$ where step sizes $\Delta a=0.005$ and $\Delta b=0.005$ have been used.

\footnotetext{
${ }^{2}$ Diverging behavior of the dynamics has not been detected by the values of the Lyapunov exponents but by an "overflow" of the solution of the jerk model while computing the Lyapunov exponents.
} 
For the regions $a<0$ or $b<0$ only diverging solutions have been found. Although this result has been derived only for one fixed set of initial conditions, it suggests that $\mathrm{JD}_{1}$ does probably not possess at all a stable attractor in the parameter regions where $a<0$ or $b<0$. Otherwise, for the chosen initial values (that are rather close to the fixed point) one would expect to find a stable attractor somewhere in these regions.

The result for the region $a>0, b>0$ is shown in Fig. 1. The different shades of grey represent different Lyapunov spectra and, therefore, different long-time dynamical behavior or attractors, respectively. White areas correspond to diverging dynamics, dark grey areas to fixed points, light grey areas to limit cycles, and black areas to points with one positive Lyapunov exponent, i.e., parameter values that lead to chaotic dynamics. One sees that with increasing $b$ the fixed point region is followed by a large region of limit cycles. Subsequently, a smaller chaotic region is present, where areas of limit cycles are embedded. Additionally, for very small $a$ and $b$ there is a tiny region with diverging dynamics that even extends into the domain where the fixed point is (locally) stable.

The corresponding route from fixed point dynamics over limit cycles to chaos is determined by the wellknown period-doubling cascade and initiated by a Hopf-bifurcation at the line $a=b$ (cf. Section 3.1). This is illustrated in Fig. 2, where a Feigenbaum-diagram for fixed $a=0.20$ and increasing $b \geqslant a$ is shown. Therefore, the large limit cycle domain, that follows the fixed point region, consists of periodic attractors with periods $2^{n}(n \in \mathbb{N})$.

Interestingly, the chaotic region develops a tongue into this domain (at $a \approx 0.54 \ldots 0.63$, $b \approx 1.05 \ldots 1.2$ ). Therefore, for fixed $a$ in this range, e.g., $a=0.60$, and increasing $b \geqslant a$ one expects a period-doubling bifurcation scenario to chaos, which then, as $b$ leaves the chaotic tongue, bifurcates backwards to a limit cycle of some period $2^{n}(n \in \mathbb{N})$ and, finally, again becomes chaotic by perioddoubling as $b$ increases further and reaches once more the chaotic region. This scenario is illustrated in Fig. 3 by the corresponding Feigenbaum-diagram. The limit cycle areas, however, that are completely enclosed by chaotic regions correspond to periodic solutions with periods that are typically not equal to $2^{n}(n \in \mathbb{N})$ and appear in Feigenbaum-diagrams as periodic windows within the chaotic range (cf. Fig. 4).

Moreover, one finds several islands of bounded dynamics in the diverging region, cf. Fig. (1), that consist of limit cycle points and points with chaotic solutions. Fig. 5 shows a Feigenbaum-plot (for $a=0.44$ ) that includes such an island (for $b \gtrsim 1.0$ ). It turns out that this island consists of two different attractors. For increasing $b$, the first one is suddenly born as strange attractor, eventually

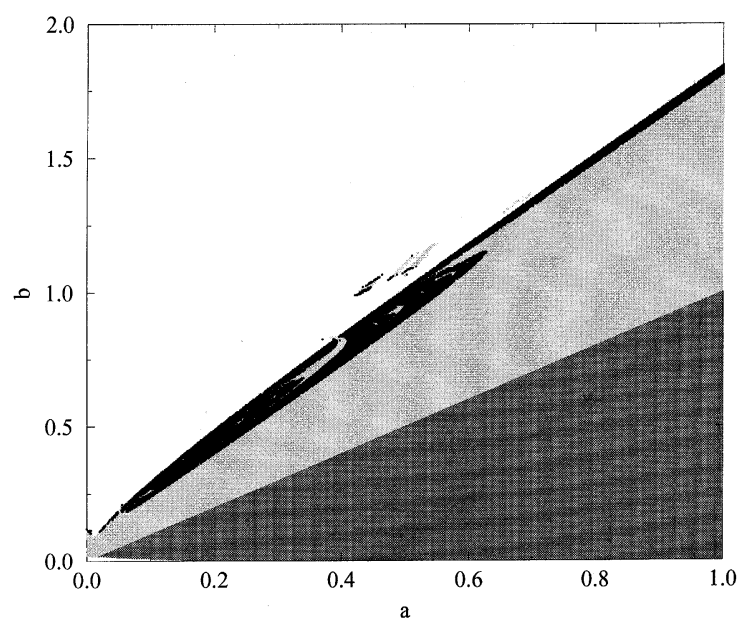

Fig. 1. Lyapunov-spectra for the jerk class $\mathrm{JD}_{1}$, Eq. (13), calculated for the initial values $x(0)=0.04, \dot{x}(0)=0.02, \ddot{x}(0)=0.0$. The different shades of grey classify the long-time dynamical behavior: white $=$ divergence, dark grey $=$ fixed point, light grey $=$ limit cycle, black $=$ strange attractor. The region of $a=1.0 \ldots 2.0$ (which has also been calculated) is not shown, because it remains qualitatively identical to the range of $a \approx 0.8 \ldots 1.0$, and is its obvious continuation. At the line $b=0$, one also finds limit cycle solutions for $a \gtrsim 0.245$. However, these limit cycles are not represented in the figure. 


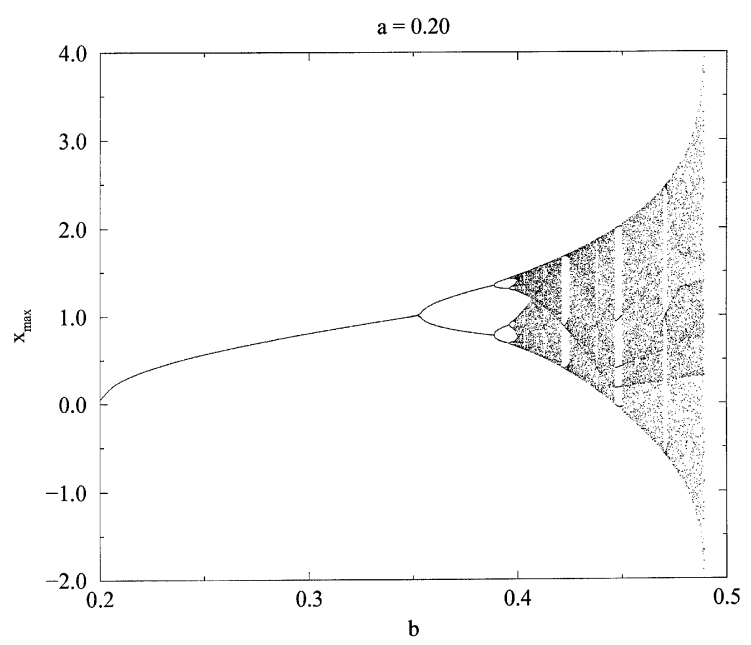

Fig. 2. Feigenbaum-diagram for the jerk class $\mathrm{JD}_{1}$ with $a=0.20$ : period-doubling cascade to chaos.

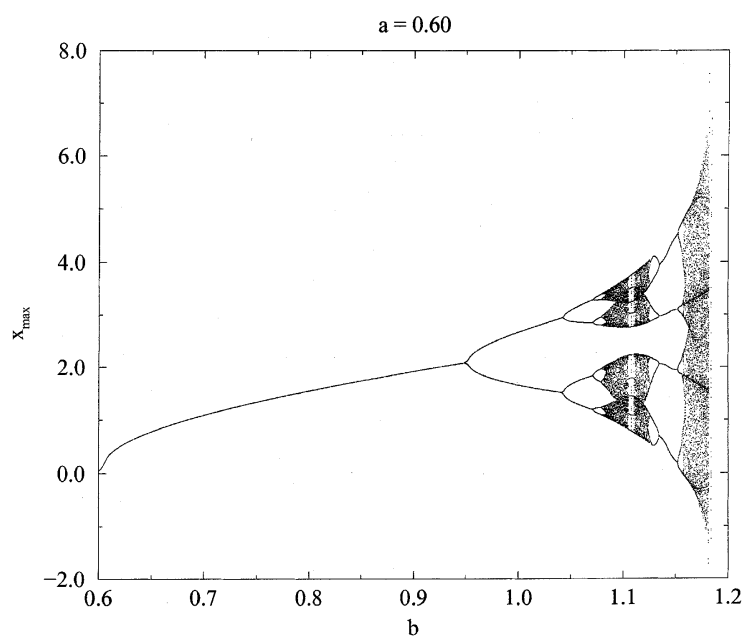

Fig. 3. Feigenbaum-diagram for the jerk class $\mathrm{JD}_{1}$ with $a=0.60$ : illustration of forward and backward bifurcations from and to chaos, respectively.

bifurcates backwards to a two-periodic limit cycle and disappears. In its place, the second attractor appears as a two-periodic limit cycle and becomes chaotic via period-doubling before disappearing again.

So far, the dependence of the dynamics on initial conditions is not taken into account. All the addressed figures are computed for the fixed initial values given above. However, using slightly altered initial values (such that they are still close to the fixed point) the qualitative appearance of Fig. 1 changes only little. Most obviously are variations of the shape and number of the islands in the diverging domain. Also the size of the tiny region with diverging dynamics at small values of $a$ and $b$ depends on the chosen initial values. This reflects the changing shape of the basin of attraction for the corresponding stable fixed point or limit cycle, respectively. Moreover, using various initial conditions, it turns out that there are coexisting stable attractors. For the above discussed island, e.g., one finds an interval of $b$, where its two attractors coexist. This situation is illustrated by the inset of Fig. 5. 


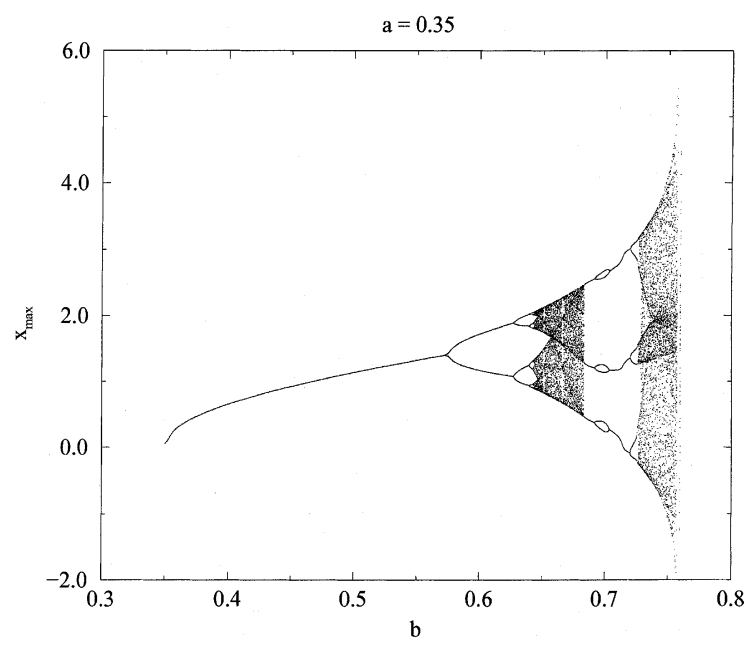

Fig. 4. Feigenbaum-diagram for the jerk class $\mathrm{JD}_{1}$ with $a=0.35$ : a large period-three window including forward and backward bifurcations to and from period-six occurs.

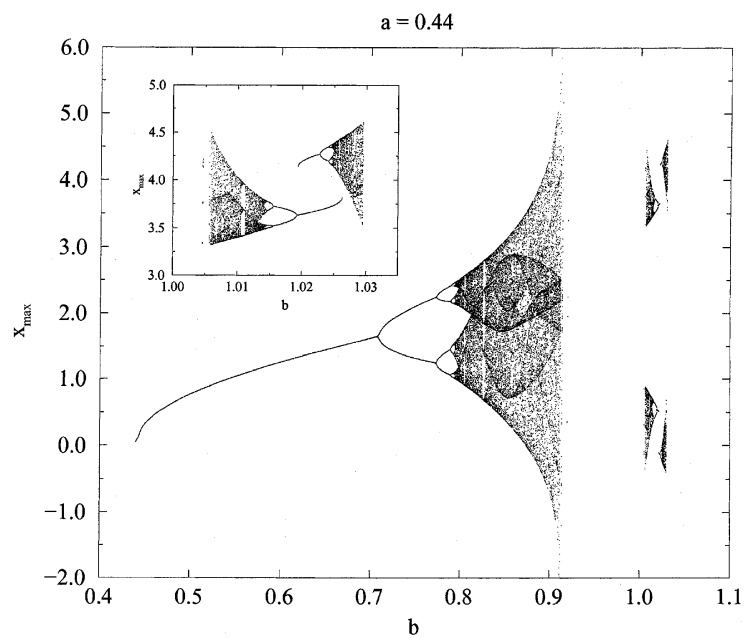

Fig. 5. Feigenbaum-diagram for the jerk class $\mathrm{JD}_{1}$ with $a=0.44$ including an island of bounded dynamics in the region of diverging dynamics. The inset shows the coexistence of stable attractors on the island (for appropriately chosen initial values).

\section{The class $\mathrm{JD}_{2}$}

Similar to the class $\mathrm{JD}_{1}$ (cf. Section 3), also the form (4) of the class $\mathrm{JD}_{2}$ has the disadvantage that the position of fixed points which exist for $k_{3}<0$ depends on the parameter value $k_{3}$ and that there are three free parameters. Therefore, we first convert $\mathrm{JD}_{2}$, Eq. (4), to a more convenient form.

\subsection{Transformation and analytical properties}

Using the transformation (9) with

$$
A=\frac{1}{2 \sqrt{\left|k_{3}\right|}}, \quad B=0, \quad C=\left|k_{1}\right| \quad\left(k_{1}, k_{3} \neq 0\right)
$$


and introducing

$$
a=-\frac{k_{2}}{k_{1}^{2}}, \quad b=-2 \frac{\sqrt{\left|k_{3}\right|}}{\left|k_{1}\right|^{3}},
$$

from (4) one obtains

$$
\dddot{x}= \pm \ddot{x}-a \dot{x}-b\left(x^{2} \mp \frac{1}{4}\right),
$$

where we have again used the notation $x$ and $t$ instead of $\bar{x}$ and $\bar{t}$.

The exclusion of the parameter values $k_{1}=0$ and $k_{3}=0$ with the above transformation again does not concern important parameter regions. For $k_{1}=0$, the system $\mathrm{JD}_{2}$ is conservative and, therefore, will not be considered here. Sprott [12] has numerically investigated some aspects of this conservative jerk model, and Maccari [36] has studied it analytically by means of an asymptotic perturbation method. For $k_{3}=0$, the dynamics of $\mathrm{JD}_{2}$, Eq. (4), is always divergent, as can be seen from the no-chaos theorem of Section 2.

With this theorem, one also finds that the dynamics of $\mathrm{JD}_{2}$, Eq. (17), always goes to infinity for the plus sign of the constant $1 / 4$. The two signs of this term are due to the above transformation, where we have to take the square root of $k_{3}$ which, for this purpose, is represented by $\pm\left|k_{3}\right|$. The minus sign of the constant term $1 / 4$ of Eq. (17) is valid for $k_{3}>0$ and the plus sign for $k_{3}<0$. On the other hand, the different signs of the $\ddot{x}$ term in Eq. (17) result from the fact that the time-scaling factor $C$ must be chosen as the modulus of $k_{1}$. The plus sign is valid for $k_{1}>0$, the minus sign for $k_{1}<0$. For the positive sign, the dynamics of $\mathrm{JD}_{2}$, Eq. (17), is divergent or located at an unstable invariant set in phase-space. This follows from its volume contraction rate (cf. Section 2), which is given by $\Lambda= \pm 1$, according to the signs of the $\ddot{x}$ term.

Taking together these findings, we infer that the functional form of $\mathrm{JD}_{2}$ with potentially bounded dynamics reads

$$
\dddot{x}=-\ddot{x}-a \dot{x}-b\left(x^{2}-\frac{1}{4}\right) .
$$

The completely unstable sets that might exist in phase-space for the plus sign of the $\ddot{x}$ term can be found by making use of the following symmetry property of $\mathrm{JD}_{2}$. Applying the transformations $t \rightarrow-t$ (which makes the completely unstable sets stable) and $b \rightarrow-b$ to the equation $\dddot{x}=+\ddot{x}-a \dot{x}-b\left(x^{2}-1 / 4\right)$ yields Eq. (18). Therefore, the stable attractors of Eq. (18) constitute the repellors of $\dddot{x}=+\ddot{x}-a \dot{x}-b\left(x^{2}-1 / 4\right)$ for signinverted $b$.

The class $\mathrm{JD}_{2}$, now represented by Eq. (18), has another interesting and useful symmetry; it is invariant under $x \rightarrow-x$ and $b \rightarrow-b$. Therefore, knowing the solution $x(t)$ of Eq. (18) for a certain value of the parameter $b$ and certain initial values, the dynamics for the corresponding sign-inverted $b$ and initial values is given by $-x(t)$.

Eq. (18) possesses the two stationary points $x^{*}= \pm 1 / 2, \dot{x}^{*}=0, \ddot{x}^{*}=0$. Analyzing their stability (cf. Section 2) leads to a characteristic equation (5) with coefficients

$$
T=-1, \quad K=-a, \quad D=\mp b .
$$

It follows that $x^{*}=+1 / 2$ is stable only for $a>0, b>0$ and $a>b$ and that it becomes unstable at the line $a=b(a, b>0)$ via a Hopf-bifurcation. Similarly, $x^{*}=-1 / 2$ is stable only for $a>0, b<0$ and $a>-b$ and becomes unstable at the line $a=-b(a>0, b<0)$ via a Hopf-bifurcation. These stability properties of the fixed points also reflect the above-mentioned symmetry of $\mathrm{JD}_{2}$, Eq. (18).

Summarizing the results of this section, we conclude that possibly interesting dynamics of the class $\mathrm{JD}_{2}$ is described by Eq. (18). Due to the discussed symmetry of this equation, we need only to consider initial values close to one of the two stationary points, e.g., $x^{*}=1 / 2, \dot{x}^{*}=0, \ddot{x}^{*}=0$. Then, the most interesting region of the $(a, b)$-parameter plane is the one for $b>a$ and positive $a$. From the studies in [29,30], we also know that this parameter region contains homoclinic orbits of the other stationary point $x^{*}=-1 / 2, \dot{x}^{*}=0$, $\ddot{x}^{*}=0$, which here is also a saddle-focus. This fact provides evidences that chaotic solutions can be observed in this parameter range $[29,30]$. 


\subsection{Numerical results}

The set of all Lyapunov exponents (cf. Section 2) of Eq. (18) is used to determine and classify the longtime dynamics of $\mathrm{JD}_{2}{ }^{2}$ and its dependence on the parameters $a$ and $b$. It has been computed for the initial values $x(t=0)=0.55, \dot{x}(t=0)=0.05$ and $\ddot{x}(t=0)=0.05$ which are close to the fixed point $x^{*}=+1 / 2$, $\dot{x}^{*}=0, \ddot{x}^{*}=0$ of $\mathrm{JD}_{2}$. The considered parameters cover a square with values of $a$ and $b$ given by $a \in[-4.0,+4.0]$ and $b \in[-4.0,+4.0]$ and step sizes $\Delta a=0.005$ and $\Delta b=0.005$.

For the parameter regions $a<0$ or $b<0$, no bounded solutions have been found. This suggests that $\mathrm{JD}_{2}$ does probably not possess at all a stable attractor in these regions. For the region $a>0$ and $b>0$ the resulting Lyapunov spectra are shown in Fig. 6. The meaning of the different shades of grey is the same as in Fig. 1. Similar to the class $\mathrm{JD}_{1}$, also for $\mathrm{JD}_{2}$ the fixed point domain is followed by a large limit cycle region and a structured chaotic region. However, the chaotic region is not present for $a \lesssim 1.5$. At $a \approx 1.5$ its boundary is formed by two tongues that reach into the limit cycle domain. For smaller parameter values, one only finds some chaotic points at $a \approx 0.8, b \approx 1.7$. Moreover, again there are islands with bounded dynamics (limit cycles and strange attractors) located within the diverging region.

To illustrate the corresponding dynamics in more detail, several Feigenbaum-diagrams for fixed $a$ and increasing $b \geqslant a$ are shown in Figs. 7-10. By virtue of Fig. 7, it becomes clear that also for $\mathrm{JD}_{2}$ the route to chaos is determined by the period-doubling cascade. Fig. 8 shows a diagram with a wide limit cycle window within the chaotic range. This window corresponds to the large limit cycle region that is embedded in the chaotic domain (cf. Fig. 6). Moreover, Fig. 8 contains one of the tiny islands with bounded dynamics that is located in the diverging region. This island consists of a chaotic attractor that, with increasing $b$, bifurcates backwards by period-halfing to a limit cycle of period 2, as can be seen from the inset of Fig. 8 .

The interesting parameter region where the two tongues of chaotic dynamics extend into the limit cycle domain is represented by Fig. 9(i). With a fixed value $a=1.6$ and increasing $b \geqslant a$, one finds two perioddoubling bifurcation scenarios to chaos with subsequent reverse bifurcations to a limit cycle of period $2^{n}$ $(n \in \mathbb{N})$. Parts of the last limit cycle range, however, consist of a new stable attractor. This sudden change of attractors can be traced back to variations of the corresponding basins of attraction with varying system parameters, whereas the initial values $x(t=0)=0.55, \dot{x}(t=0)=0.05$ and $\ddot{x}(t=0)=0.05$ are held fixed.

In Fig. 10(i), $a$ is chosen to be 0.73 and is, therefore, from the region where only a few chaotic points are present and the area with bounded dynamics looks "frayed" (cf. Fig. 6). It shows only fragments of a complete Feigenbaum-diagram. This is again due to changing boundaries of the attracted region in phasespace upon changing system parameters.

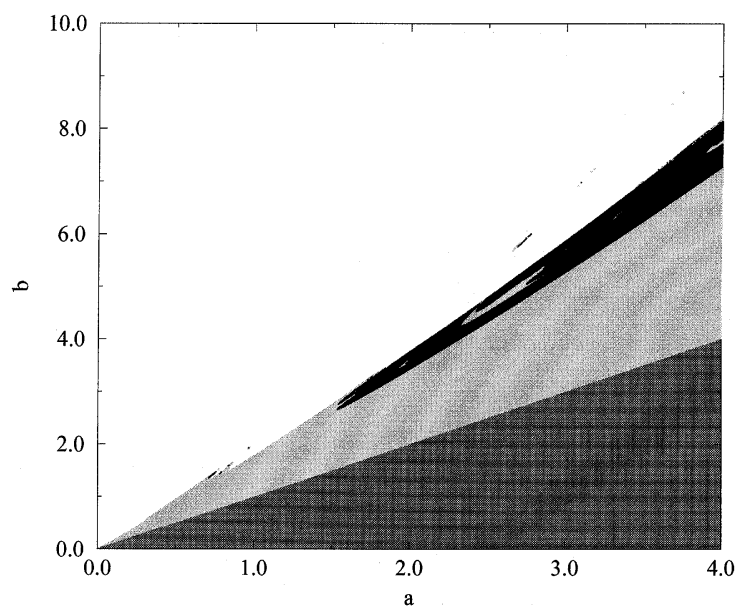

Fig. 6. Lyapunov-spectra for the jerk class $\mathrm{JD}_{2}$, Eq. (18), calculated for the initial values $x(0)=0.55, \dot{x}(0)=0.05, \ddot{x}(0)=0.05$. The different shades of grey classify the long-time dynamical behavior: white $=$ divergence, dark grey $=$ fixed point, light grey $=$ limit cycle, black $=$ strange attractor. To obtain a complete picture in the shown parameter region, computations have been performed up to a value for $b$ of +10.0 . 


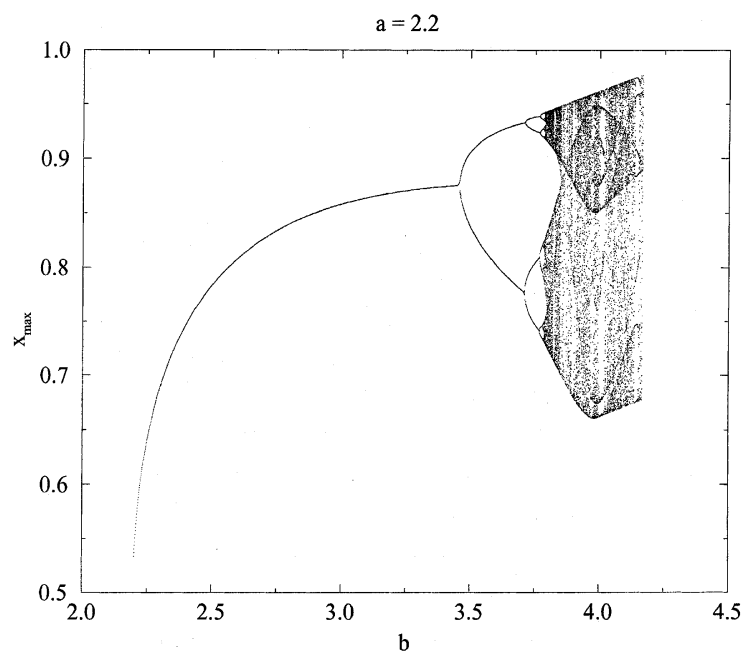

Fig. 7. Feigenbaum-diagram for the jerk class $\mathrm{JD}_{2}$ with $a=2.2$ : period-doubling cascade to chaos.

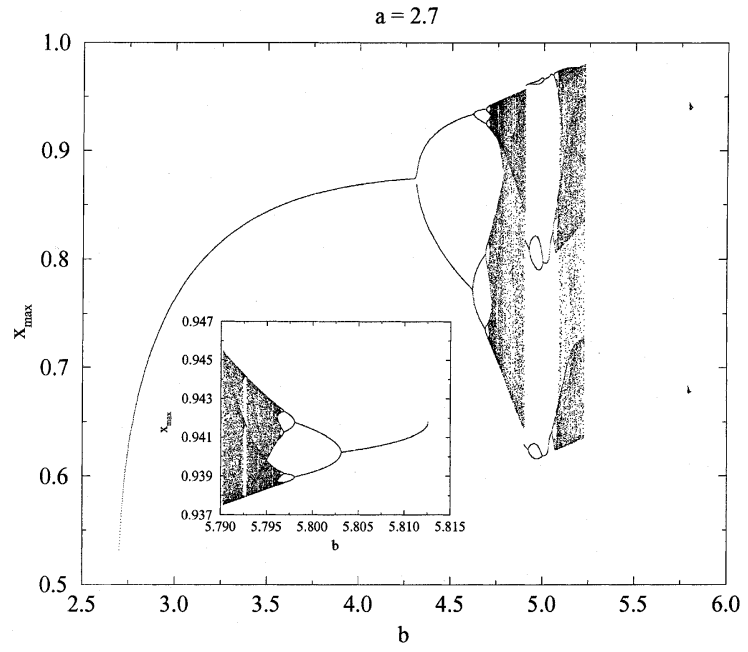

Fig. 8. Feigenbaum-diagram for the jerk class $\mathrm{JD}_{2}$ with $a=2.7$ : large period-three window including forward and backward bifurcations to and from period-six, respectively. Also an island of bounded dynamics in the region of diverging dynamics is present. The inset shows an enlargement (of the upper part) of the island attractor.

This dependence of basins of attraction on system parameters can be overcome by choosing appropriate initial values, that are still close to the fixed point $x^{*}=+1 / 2, \dot{x}^{*}=0, \ddot{x}^{*}=0$. The results are shown in Figs. 9(ii) and 10(ii). As one can see for $a=1.6$, there are coexisting stable attractors in a certain range of $b$. For $a=0.73$, it turns out that the period-doubling scenario to chaos is, in fact, broken by a diverging region and, moreover, includes infinitely many backward bifurcations (periodhalfing) that lead finally to a period-two limit cycle. Additionally, there are coexisting stable attractors for small ranges of $b$. However, for slightly varying initial conditions, the parameter regions for limit cycle or chaotic dynamics do not change qualitatively. Therefore, the main features of Fig. 6 are preserved. Mainly the number and shapes of the islands in the diverging regime vary with the choice of the initial values.

The complicated structure of Fig. 6 with islands, tongues and frayed boundaries and the underlying variety of dynamical behavior which is partly displayed by the Feigenbaum-plots of Figs. 7-10 can be related to the occurrence of homoclinic orbits of different types as is shown by Glendinning and Sparrow 

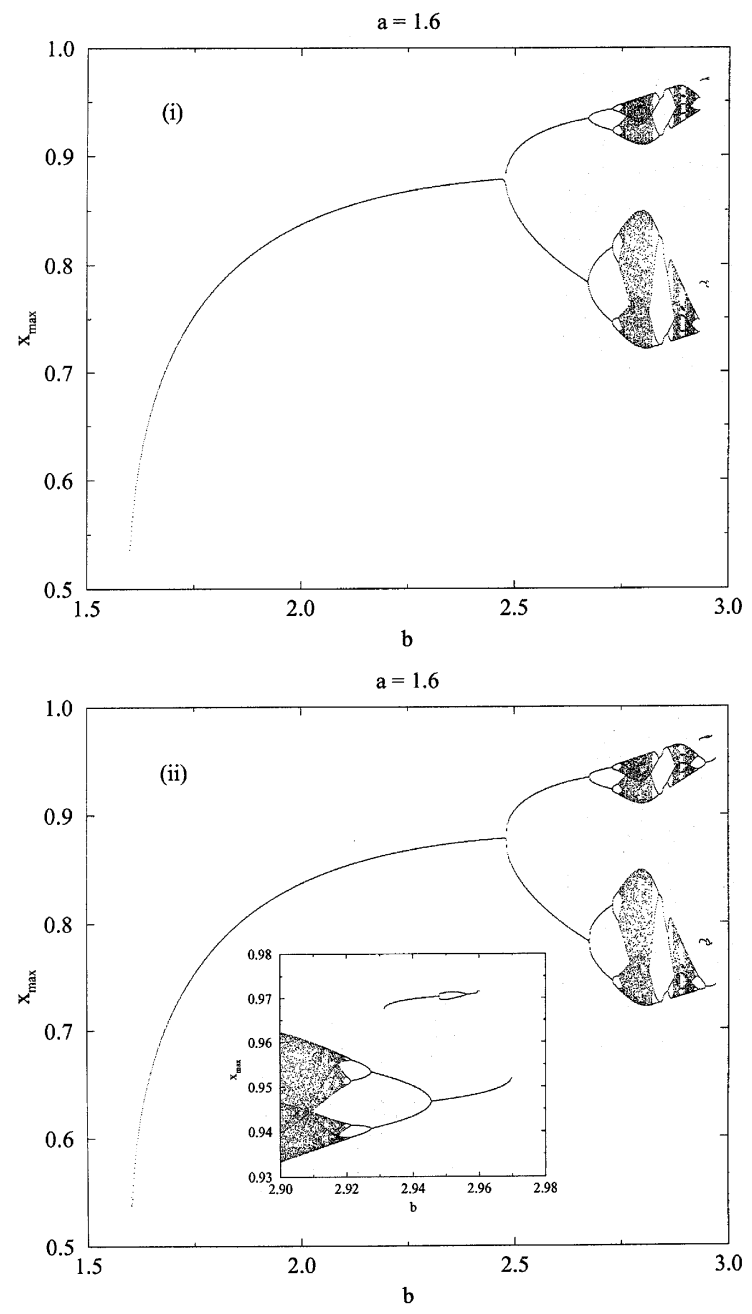

Fig. 9. Feigenbaum-diagram for the jerk class $\mathrm{JD}_{2}$ with $a=1.6$ : two successive forward and backward bifurcations to and from chaos occur that are shown: (i) for fixed initial values; (ii) for appropriately chosen initial values illustrating the coexistence of stable attractors (see inset).

[29]. Summarizing their results, we find, loosely speaking, the following. For fixed $a$, one can imagine the periodic orbit which is created via the Hopf bifurcation at $b=a$ and which is called the principal periodic orbit being destroyed by becoming a homoclinic orbit at some $b_{\mathrm{h}}>a$. In this sense, the stable periodic orbit near $a$ (i.e., $b \gtrsim a$ ) is the observable relic of a corresponding homoclinic orbit that exists for a very specific value $b=b_{\mathrm{h}}$. In between those two values $b=a$ and $b_{\mathrm{h}}$ basically two different scenarios are possible: either, the principal periodic orbit becomes unstable by a finite or infinite sequence of period-doubling bifurcations leading to a periodic orbit of the corresponding period or to chaos, respectively. Then, that sequence of bifurcations reverses itself (period halfing), eventually restabilizing the principal orbit. Such a scenario can be observed twice in Fig. 9. Or, the period-doubling sequence is terminated because a periodic orbit of higher period becomes a homoclinic orbit. Then, the reverse bifurcation sequence is started by another homoclinic orbit from that a corresponding periodic orbit arises. In between these two homoclinicities no stable behavior is observed and there appears a gap in the bifurcation diagram (cf. Fig. 10) that gives rise to the islands in the global picture of Fig. 6. Additionally to these two scenarios, in the neighborhood of all appearing homoclinic orbits several periodic solutions that emerge by saddle-node bifurcations can coexist. Such a behavior can be seen in Fig. 9 in the range $b \approx 2.925 \ldots 2.96$ and in Fig. 10 at $b \approx 1.52$. 

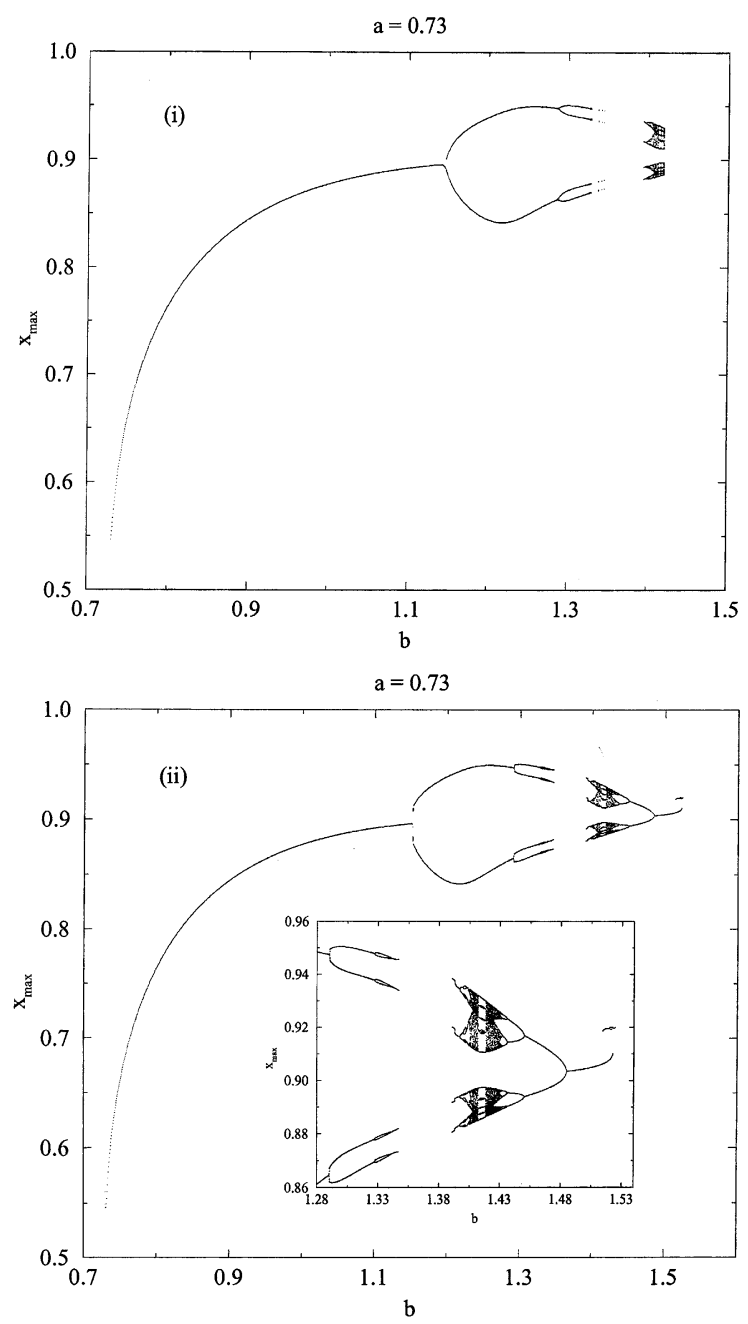

Fig. 10. Feigenbaum-diagram for the jerk class $\mathrm{JD}_{2}$ with $a=0.73$ : (i) for fixed initial values; (ii) for appropriately chosen initial values yielding additional details of the bifurcation diagram. A coexistence of stable attractors can be detected near the most right part in the inset.

\section{Discussion and conclusions}

In this paper, we investigated some aspects of the dynamical properties of the two functionally simplest polynomial classes of jerky dynamics, $\mathrm{JD}_{1}$ and $\mathrm{JD}_{2}$, that have been known to exhibit chaotic behavior for some few parameter values. As a major result, we have found for both classes that there are not only few parameters but wide ranges of parameter values that lead to chaotic long-time dynamics. Moreover, also large parameter regions are present where the long-time attractor of the dynamics consists of stable limit cycles. The route to chaos is determined by a period-doubling cascade (for appropriately varied system parameters) that is initiated by a Hopf-bifurcation. Varying the initial conditions, we also were able to detect several coexisting stable attractors. Therefore, despite their functional simplicity, the two classes $\mathrm{JD}_{1}$ and $\mathrm{JD}_{2}$ show a rich diversity of dynamical behavior which, we suggest, is not yet completely explored by our investigations.

Having taken advantage of the works of Glendinning and Sparrow [29] and Arnéodo et al. [30], we have seen that the complexity and diversity of the dynamics of $\mathrm{JD}_{2}$ is associated with the appearance of various homoclinic orbits. These homoclinic orbits are in close relation to corresponding periodic trajectories; the periodic orbit that is created from one stationary point eventually forms the homoclinic orbit of the other stationary point. Therefore, basic properties of the dynamics of $\mathrm{JD}_{2}$ likely are the result of a global 


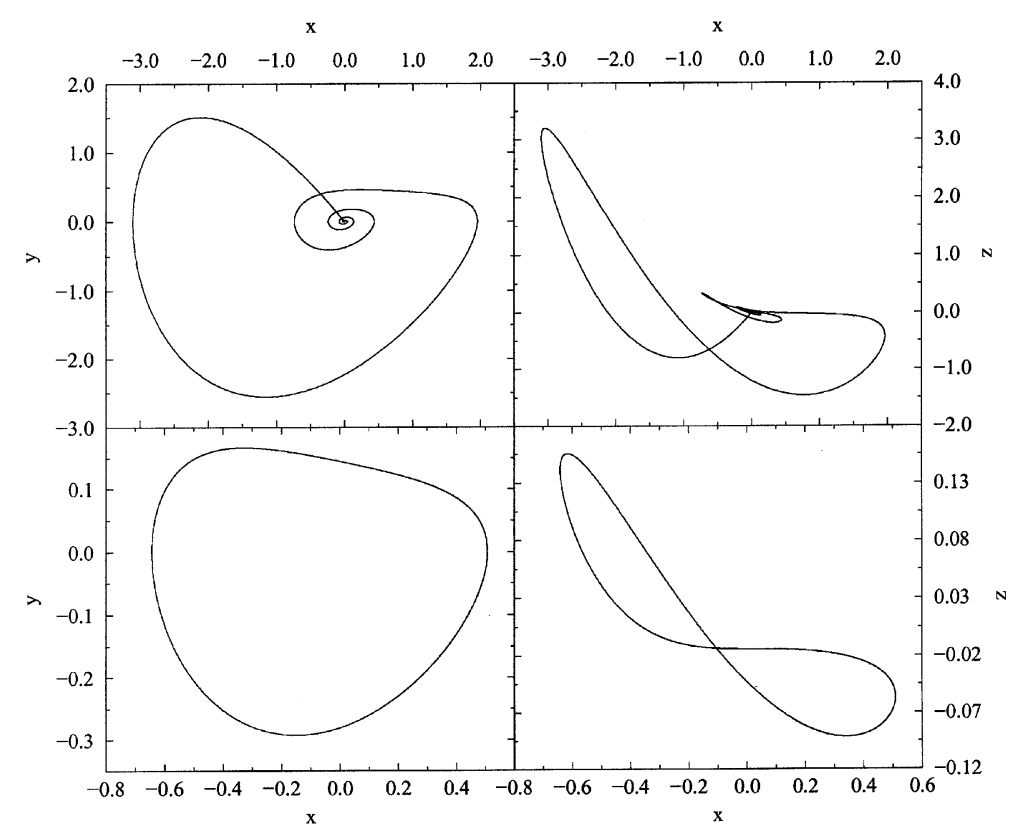

Fig. 11. Homoclinic and corresponding periodic orbit of the jerk class $\mathrm{JD}_{1}$, Eq. (13): in the upper row two projections on different planes of the phase-space of a homoclinic orbit for $a=0.1, b=0.4304863$ are depicted. The spiral around the stationary point (which is the origin) corresponds to its unstable manifold. The nearly straight part of the orbit ending at the stationary point represents its stable manifold. This homoclinic orbit is associated with the periodic orbit ( $a=0.1, b=0.15$ ) depicted in the lower row (two different projections). In the text, such a period-one orbit is called principal periodic orbit. The labels $x, y, z$ at the axes of the plots refer to the representation of a jerky dynamics as a dynamical system (cf. Eq. (2)).

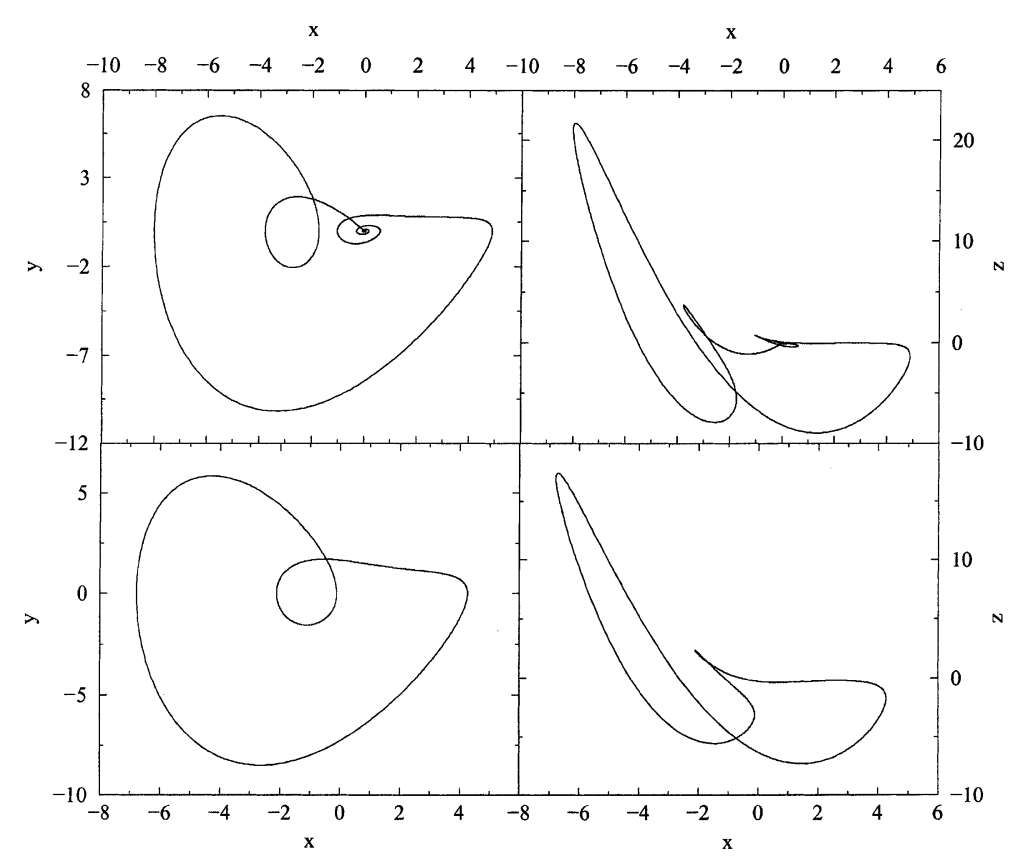

Fig. 12. Homoclinic and corresponding periodic orbit of $\mathrm{JD}_{1}$, Eq. (13): in the upper row two projections on different planes of the phase-space of a homoclinic orbit for $a=0.2, b=0.7442153$ are shown. The small spiral around the stationary point (which is the origin) corresponds to its unstable manifold. The part of the orbit ending at the stationary point represents its stable manifold. This homoclinic orbit is of another type as the one shown in Fig. 11 and corresponds to a period-two orbit which is depicted in the lower row for $a=0.44, b=1.022$ (two different projections). The labels $x, y, z$ at the axes of the plots refer to the representation of a jerky dynamics as a dynamical system (cf. Eq. (2)). 
interaction between its two fixed points [29]. Now, since our results for the classes $\mathrm{JD}_{1}$ and $\mathrm{JD}_{2}$ show qualitative similarities (islands, tongues in Figs. 1 and 6 etc.), one might expect that homoclinic orbits also play a significant role for the dynamics of $\mathrm{JD}_{1}$. In fact, with a numerical search procedure we have been able to detect homoclinic orbits of $\mathrm{JD}_{1}$; two different ones are depicted in Figs. 11 and 12 (upper parts) together with their periodic counterparts (lower parts). Hence, the dynamics of $\mathrm{JD}_{1}$ is likely determined by the interactions between the stable and unstable manifolds of the single stationary point leading, finally, to homoclinicity.

\section{Acknowledgements}

The authors acknowledge support by the "Graduiertenkolleg: Nichtlineare Probleme in Analysis, Geometrie und Physik" (Grant No. GRK 283) financed by the DFG and the state of Bavaria.

\section{References}

[1] Argyris J, Faust G, Haase M. An exploration of chaos. Amsterdam: North-Holland; 1994.

[2] Ott E. Chaos in dynamical systems. New York: Cambridge University Press; 1993.

[3] Strogatz SH. Nonlinear dynamics and chaos. Reading, MA: Addison-Wesley; 1994.

[4] Jackson EA. Perspectives of nonlinear dynamics I, II. New York: Cambridge University Press; 1991.

[5] Wiggins S. Introduction to applied nonlinear dynamical systems and chaos. New York: Springer; 1990.

[6] Guckenheimer J, Holmes P. Nonlinear oscillations, dynamical systems, and bifurcations of vector fields. New York: Springer; 1993.

[7] Drazin PG. Nonlinear systems. Cambridge: Cambridge University Press; 1992.

[8] Moon FC. Chaotic vibrations. New York: Wiley; 1987.

[9] Zhang Fu, Heidel J. Non-chaotic behavior in three-dimensional quadratic systems. Nonlinearity 1997;10:1289-303 (cf. the Erratum: Nonlinearity 1999;12:739).

[10] Heidel J, Zhang Fu. Nonchaotic behavior in three-dimensional quadratic systems II. The conservative case. Nonlinearity 1999;12:617-33.

[11] Sprott JC. Some simple chaotic flows. Phys Rev E 1994;50:R647-50.

[12] Sprott JC. Simplest dissipative chaotic flow. Phys Lett A 1997;228:271-4.

[13] Sprott JC. Some simple chaotic jerk functions. Am J Phys 1997;65:537-43.

[14] Lorenz EN. Deterministic nonperiodic flow. J Atmos Sci 1963;20:130-41.

[15] Rössler OE. An equation for continuous chaos. Phys Lett A 1976;57:397-8.

[16] Eichhorn R, Linz SJ, Hänggi P. Transformations of nonlinear dynamical systems to jerky motion and its application to minimal chaotic flows. Phys Rev E 1998;58:7151-64.

[17] von Baeyer HC. All shook up. The Sci 1998;38(1):12-4.

[18] Gottlieb HPW. Qestion 38. What is the simplest jerk function that gives chaos? Am J Phys 1996;64:525.

[19] Linz SJ. Nonlinear dynamical models and jerky motion. Am J Phys 1997;65:523-6.

[20] Sprott JC. Some simple chaotic jerk functions. Am J Phys 1997;65:537-43.

[21] Linz SJ. Newtonian jerky dynamics: Some general properties. Am J Phys 1998;66:1109-14.

[22] Gottlieb HPW. Simple nonlinear jerk functions with periodic solutions. Am J Phys 1998;66:903-6.

[23] Linz SJ, Sprott JC. Elementary chaotic flow. Phys Lett A 1999;259:240-5.

[24] Malasoma JM. What is the simplest dissipative chaotic jerk equation which is parity invariant?. Phys Lett A 2000;264:383-9.

[25] Perko L. Differential equations and dynamical sytems. New York: Springer; 1996.

[26] Rössler OE. Continuous chaos - four prototype equations. Ann N Y Acad Sci 1979;316:376-92.

[27] Coullet P, Tresser C, Arnéodo A. Transition to stochasticity for a class of forced oscillators. Phys Lett A 1979;72:268-70.

[28] Arnéodo A, Coullet P, Tresser C. Oscillators with chaotic behavior: an illustration of a theorem by Shil'nikov. J Stat Phys 1982;27:171-82.

[29] Glendinning P, Sparrow C. Local and global behavior near homoclinic orbits. J Stat Phys 1984;35:645-96.

[30] Arnéodo A, Coullet PH, Spiegel EA, Tresser C. Asymptotic chaos. Physica D 1985;14:327-47.

[31] Bronstein IN, Semendjajew KA. Taschenbuch der Mathematik. Harri Deutsch: Frankfurt/Main; 1987.

[32] Eckmann JP, Ruelle D. Ergodic theory of chaos and strange attractors. Rev Mod Phys 1985;57:617-56.

[33] Campanino M. Two remarks on the computer study of differentiable dynamical systems. Comm Math Phys 1980;74:15-20.

[34] Shimada I, Nagashima T. A numerical approach to ergodic problem of dissipative dynamical systems. Prog Theo Phys 1979;61:1605-16.

[35] Benettin G, Galgani L, Giorgilli A, Strelcyn JM. Lyapunov characteristic exponents for smooth dynamical systems and for Hamiltonian systems; a method for computing all of them Part I. Theory. Meccanica 1980;15:9-20.

[36] Maccari A. The non-local oscillator. Nuovo Cimento 1996;111:917-30. 